\title{
Ultrasonic particle sizing in aqueous suspensions of solid particles of unknown density
}

\author{
Raied S. Al-Lashi ${ }^{1, a)}$ and Richard E. Challis ${ }^{2}$ \\ ${ }^{1}$ School of Food Science and Nutrition, Faculty of Mathematics and Physical Sciences, University of Leeds, \\ Leeds, United Kingdom \\ ${ }^{2}$ Electrical Systems and Optics Division, Faculty of Engineering, University of Nottingham, Nottingham, \\ United Kingdom
}

(Received 20 November 2014; revised 5 June 2015; accepted 20 July 2015; published online 20 August 2015)

Estimates of particle size distributions (PSDs) in solid-in-liquid suspensions can be made on the basis of measurements of ultrasonic wave attenuation combined with a mathematical propagation model, which typically requires seven physical parameters to describe each phase of the mixture. The estimation process is insensitive to all of these except the density of the solid particles, which may not be known or difficult to measure. This paper proposes that an unknown density value is incorporated into the sizing computation as a free variable. It is shown that this leads to an accurate estimate of PSD, as well as the unknown density. (C) 2015 Acoustical Society of America.

[http://dx.doi.org/10.1121/1.4927694]

[NAG]

Pages: 1023-1029

\section{INTRODUCTION}

A colloidal suspension consists of small solid particles dispersed in a surrounding liquid continuum with particle sizes in the range $10 \mathrm{~nm}$ to $100 \mu \mathrm{m} .{ }^{1}$ The particle size distribution (PSD) is an important property of a suspension because it determines the stability and shelf life of the material, as well as its quality and functionality when in final product form. The PSD can be accurately estimated using measurements of ultrasonic wave attenuation across a frequency band together with an appropriate wave propagation model, the most commonly used being that due to Epstein and Carhart $^{2}$ and Allegra and Hawley, ${ }^{3}$ known as ECAH. The technique is currently included in international standards, such as Refs. 4 and 5. It has the major advantage that it can be applied on-line in a plant although applications to real industrial processes have been slow to evolve. We believe that one reason for this is the existence of uncertainties associated with the method and this paper addresses one significant uncertainty-that associated with the disperse phase density.

The ECAH model has as its input seven physical constants to describe each phase of the mixture-the solid particulate component and the liquid continuum. Whilst for many materials these data are available from standard handbooks ${ }^{6,7}$ there remain many materials for which such data are either not known or are too costly or too dangerous to measure. In a recent publication, ${ }^{8}$ we have challenged the assumption that all of the 14 inputs to the ECAH model are required to be known accurately; we demonstrated that the wave attenuation is relatively insensitive to all of the physical constants except the density of both phases, although it is that of the solid phase which is generally unknown. There is also uncertainty as to what is

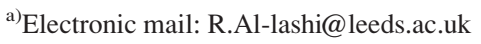

the effective viscosity of the continuous phase surrounding the particles due to interactions between mode-converted evanescent shear waves scattered from suspended particles in proximity. In Ref. 9, we have shown that a pragmatic solution to this uncertainty where solids concentration exceeds $2 \% \mathrm{v} / \mathrm{v}$ (volume of the solid/total volume of the mixture) is to use the Happel model for viscosity in suspensions ${ }^{10-12}$ in place of the viscosity of water in the ECAH model.

The purpose of this paper is to propose a technique by which an unknown density can form part of the particle sizing process, thus, eliminating the problem of expensive or hazardous measurements. We outline the particle sizing process and the degree of uncertainty associated with erroneous values for the physical constants of the disperse phase. Experiments on PSD estimation were done on three different materials-a monodisperse suspension of silica particles (Ludox), a polydisperse aqueous suspension of magnesium oxide particles (Versamag), and an aqueous suspension of glass beads. In each case, the particle size estimations were done twice, once with an assumed "true" value for solid phase density and then with the value of density used as a free variable in the PSD computation. The results are compared to the specified particle size for the Ludox, and for the Versamag and glass beads, to benchmark size data obtained from optical scattering measurements.

\section{PSD ESTMATION BY ULTRASOUND}

In a typical PSD estimation, ultrasonic wave attenuation is measured as a broad frequency spectrum between $1 \mathrm{MHz}$ and (typically) $30 \mathrm{MHz}$, although $100 \mathrm{MHz}$ is possible. In principle, the corresponding dispersion in phase velocity with respect to frequency could be used in PSD estimation, but this is generally discounted in that the attenuation is more sensitive to particle size and is certainly much easier to 
measure with reasonable accuracy. A mathematical model of ultrasonic wave propagation is used to simulate the measured attenuation spectrum, the most commonly used being the ECAH formulation. ${ }^{2,3} \mathrm{We}$ use an extended form of this model, which takes account of multiple scattering of compression waves between adjacent particles in the mixture; it is based on the work of Lloyd and Berry, ${ }^{13}$ and is reviewed in Ref. 1. The original ECAH model was limited to monodisperse mixtures, but we have employed a further extension to enable the model to incorporate an arbitrary number of size bins, $J$; see Ref. 14. The complex wave number in the mixture becomes

$$
\frac{\beta^{2}}{k_{c}^{2}}=1+\sum_{j=1}^{J}\left[\frac{3 \phi_{j}}{i k_{c}^{3} r_{j}^{3}}\left(A_{0 j}+3 A_{1 j}+5 A_{2 j}\right)-\frac{27 \phi_{j}^{2}}{k_{c}^{6} r_{j}^{6}}\left(A_{0 j} A_{1 j}+5 A_{1 j} A_{2 j}\right)-\frac{54 \phi_{j}^{2}}{k_{c}^{6} r_{j}^{6}}\left(A_{1 j}^{2}+\frac{5}{3} A_{0 j} A_{1 j}+3 A_{1 j} A_{2 j}+\frac{115}{21} A_{2 j}^{2}\right)\right]
$$

Here, $\beta$ is the complex wave number in the mixture, $k_{c}$ is the compression wave number in the continuous phase, $\phi_{j}$ is the volume fraction of particles of radius, $r_{j}, j$ identifies the size bin, and $J$ is the total number of size bins. $A_{0 j}, A_{1 j}$, and $A_{2 j}$ are the partial wave amplitude coefficients, which pertain to size bin, $j$. On the basis that elemental sinusoids are denoted $e^{+i \omega t}$, the complex wave number is

$$
\beta=\frac{\omega}{c(\omega)}-i \alpha(\omega)
$$

where $\omega$ is angular frequency, $c(\omega)$ is phase velocity and $\alpha(\omega)$ is the amplitude attenuation coefficient.

It is well known that the ECAH model breaks down when the concentration of dispersed solid particles exceeds a certain limit, which can be as low as $2 \% \mathrm{v} / \mathrm{v}$. The model predicts higher attenuation values than are found by measurement and the error increases with particle concentration; this is thought to be due to the overlap of mode-converted shear waves, which emanate from the scattering particles; see Ref. 1. In Ref. 9, we have shown that the problem can be at least partially overcome when the assumed viscosity of the continuous phase, which is input to the model, is modified from that of water to a new equivalent value derived from classical models of viscosity in particulate suspensions that have been proposed in studies of flow or sedimentation phenomena. The best, but not perfect, viscosity model was found to be that due to Happel et al. ${ }^{10-12}$ where the effective viscosity was

$$
\eta_{\text {eff }}=\frac{2+\frac{4}{3} \phi^{5 / 3}}{2-3 \phi^{1 / 3}+3 \phi^{5 / 3}-2 \phi^{2}} \eta_{\text {water }}
$$

This formulation gives simulations of attenuation to within, approximately, $\pm 15 \%$ of measured values; this is to be compared with ECAH simulations in which the viscosity of water is used and which overpredict attenuation values by as much as $300 \%$. Notwithstanding the still significant error, we have used the Happel viscosity for concentrations $>2 \%$ $\mathrm{v} / \mathrm{v}$, it being the best available at the present time. We note that is neither a function of particle size nor of frequency.

As well as viscosity and density, the model has as its inputs the other physical properties of both phases of the suspension and a candidate PSD function, which is adapted systematically until best match is obtained between simulated and measured attenuation data in a least-squared-error sense, the Marquardt algorithm ${ }^{15,16}$ being commonly used for this. The candidate PSD functions are mostly log-normal in particle size and are defined by a central size value (median or mean) and a standard deviation as a dimensionless width parameter. The use of simple two-parameter functions yields a unimodal estimate of PSD which may approximate a more complex distribution. This is in contrast to optical sizing techniques, such as the Mastersizer instrument (Malvern Instruments Ltd., Malvern, UK), which yields a parameter-free distribution in which more complex PSD shapes are observable.

In this work, we propose to add an unknown solid phase density as a third parameter in the model adaption stage of the sizing process.

\section{PARAMETER UNCERTAINTY}

It is customary to assume that reliable values for the physical properties of a suspension are required for accurate simulations using the ECAH model ${ }^{1}$ but, as noted in the Introduction, some or all of these parameters may not be available. We have investigated the effect of uncertainty in parameter values for silica suspensions in Ref. 8 and here we repeat the exercise for Versamag. The ultrasonic attenuation at $10 \mathrm{MHz}$ in a $6.54 \% \mathrm{v} / \mathrm{v}$ aqueous suspension of Versamag was simulated using the extended ECAH model and the physical properties given in Table I. The suspension was, at this point, assumed to be monodisperse with all suspended particle diameters set to $1.8 \mu \mathrm{m}$. Each of the physical properties of the Versamag was systematically changed between an assumed central true value $\pm 50 \%$ in steps of $10 \%$. The results are given in Table II, from which it will be clear that even at a variation of $50 \%$, the change in attenuation ranged between $0.0000 \%$ and $0.54 \%$. Thus, any reasonable guess of these physical property values will have little effect on the attenuation calculation. By contrast, Fig. 1 shows the effect on attenuation values at $10 \mathrm{MHz}$ for variations in solid phase density up to $\pm 20 \%$ about a notional true value. The calculations have been done for monodisperse suspensions, as well as polydisperse ones with standard deviations of $0.25,0.5$, 
TABLE I. Physical parameters used in ECAH simulation. There are gaps in the table where some properties of Versamag and Glass Beads were unknown; the corresponding properties of silica were used in these cases.

\begin{tabular}{|c|c|c|c|c|}
\hline $\begin{array}{l}\text { Parameter } \\
\text { (SI units) }\end{array}$ & $\begin{array}{l}\text { Magnesium } \\
\text { hydroxide } \\
\text { (Versamag) }\end{array}$ & Silica & Glass beads & Water $\left(25^{\circ} \mathrm{C}\right)$ \\
\hline$c\left(\mathrm{~ms}^{-1}\right)$ & & $5968^{b}$ & & $1497^{\mathrm{c}}$ \\
\hline$\rho\left(\mathrm{kg} \mathrm{m}^{-3}\right)$ & $2370^{d}$ & $2185^{\mathrm{b}}$ & 2500 (Ref. 1) & $977^{\mathrm{c}}$ \\
\hline$\mu\left(\mathrm{Nm}^{-2}\right)$ & & $3.09 \mathrm{e}^{10 \mathrm{~b}}$ & & \\
\hline$M(\mathrm{Pas})$ & & $8.441 \mathrm{e}^{10 \mathrm{f}}$ & & \\
\hline$\eta(\mathrm{Pa} \mathrm{s})$ & & & & $8.91 \mathrm{e}^{-4 \mathrm{~d}}$ \\
\hline$\kappa\left(\mathrm{W} \mathrm{m}^{-1} \mathrm{~K}^{-1}\right)$ & & $1.6^{\mathrm{b}}$ & & $0.595^{\mathrm{d}}$ \\
\hline$C_{p}\left(\mathrm{~J} \mathrm{~kg}^{-1} \mathrm{~K}^{-1}\right)$ & $1320.31^{\mathrm{d}}$ & $729^{\mathrm{b}}$ & & $4179^{b}$ \\
\hline$\alpha / f^{2}\left(\mathrm{Nps}^{2} \mathrm{~m}^{-1}\right)$ & & $2.6 \mathrm{e}^{-22 \mathrm{a}}$ & & $2.3 \mathrm{e}^{-14 \mathrm{e}}$ \\
\hline$\beta_{T}\left(\mathrm{~K}^{-1}\right)$ & & $1.35 \mathrm{e}^{-6 \mathrm{~b}}$ & & $2.1 \mathrm{e}^{-4 b}$ \\
\hline
\end{tabular}

${ }^{\mathrm{a}}$ Experimental measurement.

${ }^{\mathrm{b}}$ Kaye and Laby (Ref. 6).

${ }^{\mathrm{c}}$ Del Grosso (Ref. 18).

${ }^{\mathrm{d}}$ CRC Handbook of Chemistry and Physics (Ref. 7).

${ }^{\text {e }}$ Smith (Ref. 19).

${ }^{\mathrm{f}}$ Value calculated from $c$ and $\rho$.

0.75, and 1.5. In all cases, the variation in attenuation is approximately twice the variation in density value.

\section{EXPERIMENTS}

The experimental apparatus is sketched in Fig. 2. The fluid circuit consisted of a reservoir containing the test suspension, an ultrasonic measuring cell (the flow cell), and a pump that maintained the solid particles in suspension by circulating the suspension from the bottom of the reservoir, through the flow cell, and back to the reservoir. The flow cell had two pairs of coaxially aligned ultrasonic transducers-transmitters and receivers - set at a gauge length of $6 \mathrm{~mm}$; the centre frequencies of the transducer pairs were $10 \mathrm{MHz}$ and $25 \mathrm{MHz}$. We have shown in Ref. 17 that errors in attenuation measurements are minimised when the total measured attenuation is around

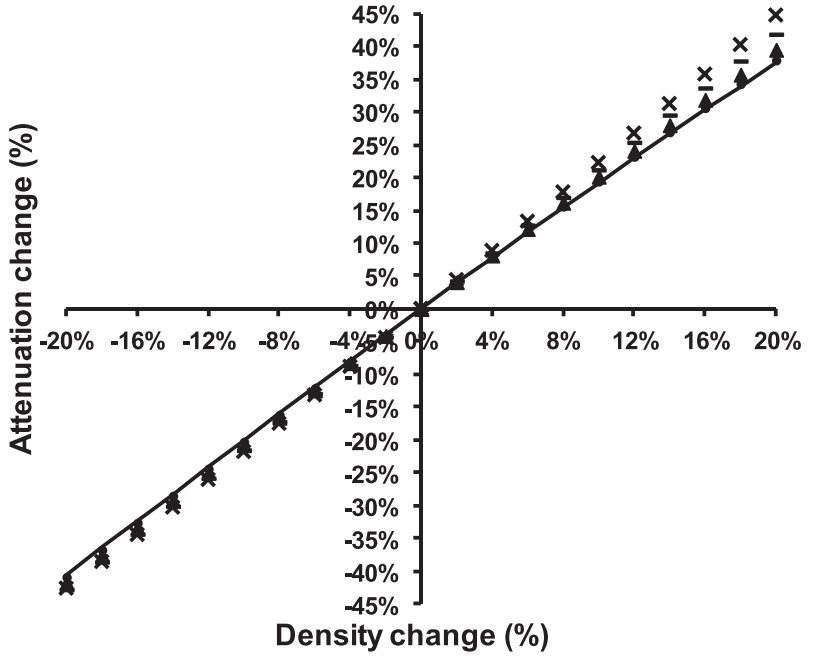

FIG. 1. Calculated attenuation sensitivity to density change for aqueous suspensions of Versamag particles of concentration $6.54 \% \mathrm{v} / \mathrm{v}$ and distributed as log-normal in volume. The solid line represents the monodisperse case $\mu=1.8 \mu \mathrm{m}$ (median diameter), the other lines are for polydisperse mixtures with $\mu=1.8 \mu \mathrm{m}$ (median diameter), and $\sigma=0.25$ (closed circles), $\sigma=0.5$ (closed triangles), $\sigma=0.75$ (dashes), and $\sigma=1.5$ (crosses).

$1 \mathrm{~Np}$. The $6 \mathrm{~mm}$ spacing matches this condition for the Versamag suspension at $\sim 10 \mathrm{MHz}$; a shorter spacing of $2.5 \mathrm{~mm}$ would be optimum for the $25 \mathrm{MHz}$ transducer, but this was impractical due to the risk of clogging of the suspended particles in the gap between the two transducers. The attenuation spectra obtained from the two pairs of transducers were combined by fitting a third order polynomial across the combined data from the two pairs. This procedure gave an effective bandwidth from $\sim 4 \mathrm{MHz}$ up to $30 \mathrm{MHz}$; it also smoothed the undulations due to electronic noise in the raw data; see Fig. 3. The smoothed attenuation data were used in the sizing computations, which used 384 frequency points equi-spaced between $0.0781 \mathrm{MHz}$ and $30 \mathrm{MHz}$. The Versamag suspension was at a concentration of $6.54 \% \mathrm{v} / \mathrm{v}$. Its PSD as measured by Mastersizer (Malvern Instruments Ltd., Malvern, UK) is shown on Fig. 4, the instrument returning a median

TABLE II. Sensitivity of attenuation at $10 \mathrm{MHz}$ in response to changes in the physical properties of a $6.54 \%$ v/v aqueous suspension of Versamag: Compression modulus $(M)$, shear modulus $(\mu)$, thermal conductivity $(\kappa)$, specific heat $\left(C_{p}\right)$, attenuation coefficient of the continuous phase (water) expressed as $\left(\alpha / f^{2}\right)$, and thermal expansion coefficient $\left(\beta_{T}\right)$. The first column in the table gives the \% change in any given physical property $(X$, say). The right-hand six columns give the corresponding change in attenuation, expressed as a differential as a function of $X$. The median diameter is $1.8 \mu \mathrm{m}$ and the standard deviation is 1.5 .

\begin{tabular}{|c|c|c|c|c|c|c|}
\hline $\begin{array}{l}\text { Change in } X \\
(\%)\end{array}$ & $\frac{\Delta \alpha}{\alpha}(M)$ & $\frac{\Delta \alpha}{\alpha}(\mu)$ & $\frac{\Delta \alpha}{\alpha}(\kappa)$ & $\frac{\Delta \alpha}{\alpha}\left(C_{p}\right)$ & $\frac{\Delta \alpha}{\alpha}\left(\alpha / f^{2}\right)$ & $\frac{\Delta \alpha}{\alpha}\left(\beta_{T}\right)$ \\
\hline 0 & $0.000 \%$ & $0.00 \%$ & $0.000 \%$ & $0.00 \%$ & $0.0000 \%$ & $0.0000 \%$ \\
\hline+10 & $0.010 \%$ & $-0.07 \%$ & $0.001 \%$ & $0.03 \%$ & $0.0000 \%$ & $-0.0012 \%$ \\
\hline+20 & $0.011 \%$ & $-0.14 \%$ & $0.002 \%$ & $0.06 \%$ & $0.0000 \%$ & $-0.0024 \%$ \\
\hline+30 & $0.009 \%$ & $-0.24 \%$ & $0.003 \%$ & $0.09 \%$ & $0.0000 \%$ & $-0.0037 \%$ \\
\hline+40 & $0.007 \%$ & $-0.37 \%$ & $0.003 \%$ & $0.12 \%$ & $0.0000 \%$ & $-0.0049 \%$ \\
\hline+50 & $0.004 \%$ & $-0.54 \%$ & $0.003 \%$ & $0.14 \%$ & $0.0000 \%$ & $-0.0061 \%$ \\
\hline-10 & $0.053 \%$ & $0.06 \%$ & $-0.001 \%$ & $-0.03 \%$ & $0.0000 \%$ & $0.0012 \%$ \\
\hline-20 & $0.035 \%$ & $0.11 \%$ & $-0.004 \%$ & $-0.07 \%$ & $0.0000 \%$ & $0.0024 \%$ \\
\hline-30 & $0.034 \%$ & $0.16 \%$ & $-0.007 \%$ & $-0.10 \%$ & $0.0000 \%$ & $0.0037 \%$ \\
\hline-40 & $0.035 \%$ & $0.23 \%$ & $-0.011 \%$ & $-0.14 \%$ & $0.0000 \%$ & $0.0049 \%$ \\
\hline-50 & $0.037 \%$ & $0.35 \%$ & $-0.017 \%$ & $-0.18 \%$ & $0.0000 \%$ & $0.0062 \%$ \\
\hline
\end{tabular}




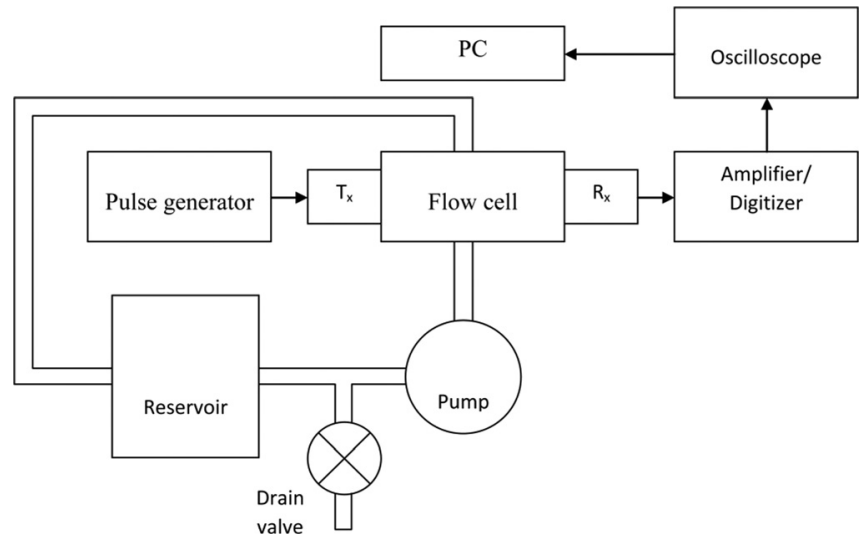

FIG. 2. Schematic representation of the experimental setup. $T_{x}$ is the transmitting transducer and $R_{x}$ is the receiving transducer.

size of $1.8 \mu \mathrm{m}$ with a standard deviation of 1.5 . The distribution appears to be bi-modal, but will be approximated to a mono-modal distribution in the ultrasonic sizing exercise.

\section{COMPUTATIONS AND RESULTS}

\section{A. Monodisperse case}

The single suspended particle size in the Ludox suspensions was calculated using an assumed fixed density of $2185 \mathrm{~kg} \mathrm{~m}^{-3}$ and also allowing the density value to be a free variable whose final value is returned from the sizing computation. The results are given in Table III, from which it will be clear that accuracy of the free-density result is commensurable with that of the fixed-density result. The variation in the fitted density with respect to the true density value is within $\pm 3.3 \%$.

\section{B. Polydisperse case 1: Versamag suspensions}

On the basis of the attenuation data of Fig. 3, the PSD of the Versamag suspension was estimated using the extended ECAH propagation model with the Happel formulation for the viscosity of the continuous phase. The physical properties for both phases are given in Table I. The sizing computation was initially done using the nominal density of Versamag of $2370 \mathrm{~kg} \mathrm{~m}^{-3}$ and then with density as a free

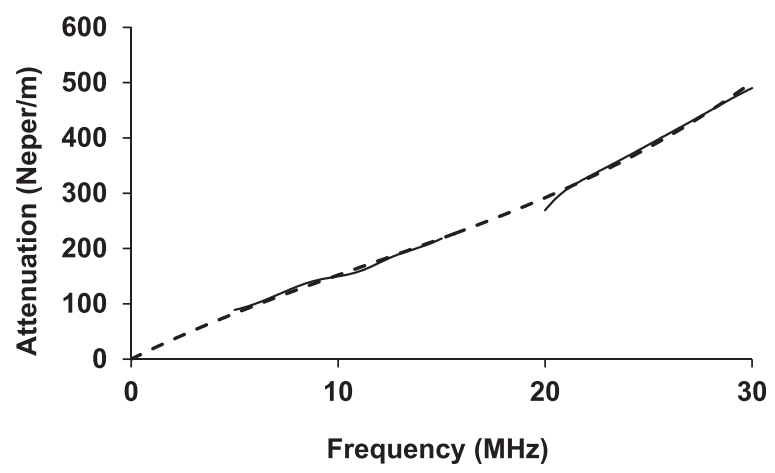

FIG. 3. Attenuation spectra of a $6.54 \% \mathrm{v} / \mathrm{v}$ aqueous suspensions of Versamag obtained from fitting a third order polynomial (dashed line) to the measured attenuation (solid lines).

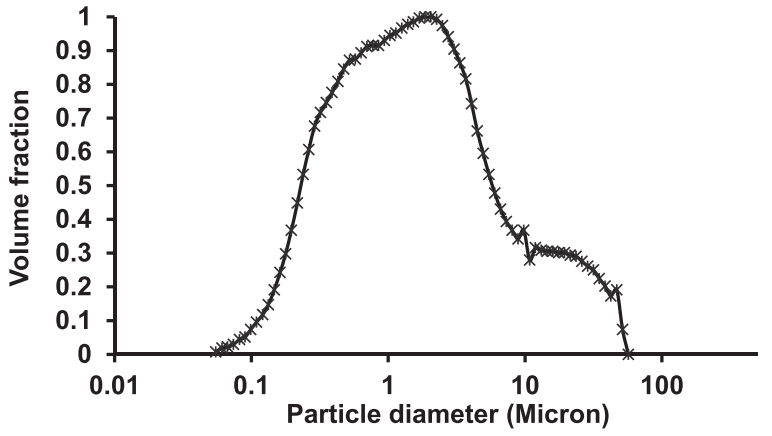

FIG. 4. PSD of $6.54 \%$ v/v Versamag sample obtained with the Mastersizer (Malvern Instruments Ltd., Malvern, UK) instrument. The median size is $1.8 \mu \mathrm{m}$ and the standard deviation is 1.5 .

TABLE III. Particle size estimates $(\mu)$ for $30 \mathrm{~nm}$ diameter silica particle suspensions (Ludox) at three concentrations ( $\phi \% \mathrm{v} / \mathrm{v})$, using for $\mu_{1}$, a fixed density value of $2185 \mathrm{~kg} \mathrm{~m}^{-3}$, and for $\mu_{2}$, allowing density to be a free variable in the PSD calculation. The adapted density value is given in column 4 , and its relative difference with respect to the "true" value in column 5.

\begin{tabular}{lcccc}
\hline \hline$\phi(\%)$ & $\mu_{1}(\mathrm{~nm})$ & $\mu_{2}(\mathrm{~nm})$ & $\rho\left(\mathrm{kg} / \mathrm{m}^{3}\right)$ & $\begin{array}{c}\text { Percentage } \\
\text { change in density }(\%)\end{array}$ \\
\hline 3.25 & 31.2 & 29.0 & 2257 & $3.3 \%$ \\
5.99 & 29.6 & 28.9 & 2197 & $0.55 \%$ \\
8.22 & 28.7 & 30.0 & 2127 & $-2.65 \%$ \\
\hline \hline
\end{tabular}

TABLE IV. PSD parameters of the Versamag sample obtained with the Mastersizer (Malvern Instruments Ltd., Malvern, UK), and ultrasonically with either a fixed value of density or with density as a free variable in the fitting process.

\begin{tabular}{lccc}
\hline \hline PSD type & Median $(\mu \mathrm{m})$ & Standard deviation & Density $\left(\mathrm{kg} \mathrm{m}^{-3}\right)$ \\
\hline Mastersizer & 1.8 & 1.5 & $\mathrm{n} / \mathrm{a}^{\mathrm{a}}$ \\
Fixed density & 1.8 & 1.4 & 2370 \\
Free density & 1.6 & 1.4 & 2346 \\
\hline \hline
\end{tabular}

an/a: Not applicable.

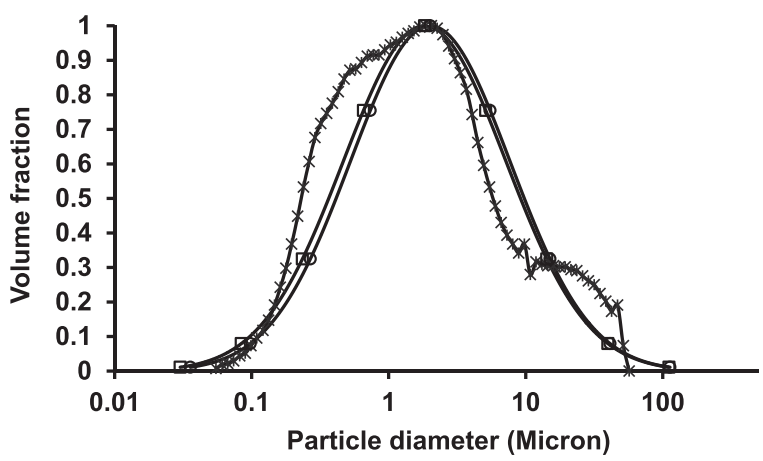

FIG. 5. Comparison of the PSD of Versamag sample obtained by the Mastersizer (Malvern Instruments Ltd., Malvern, UK) instrument (crosses) with those obtained ultrasonically: The squares give the result when the "true" density was used in the fitting, and the circles give the result when the solid phase density is allowed to be a free variable in the fitting process. 


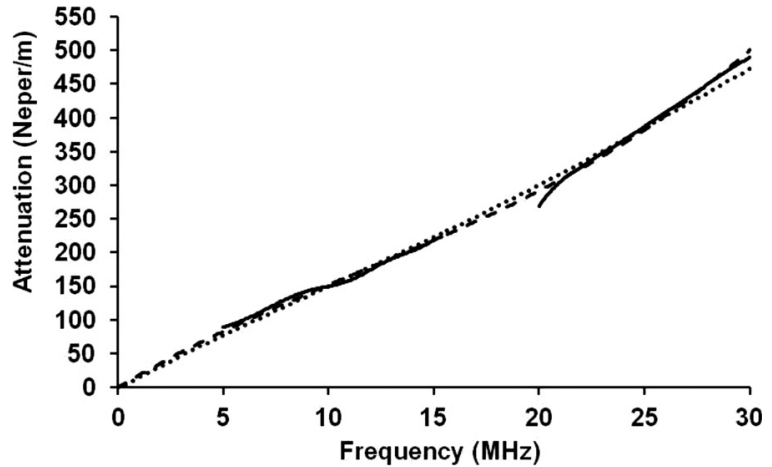

FIG. 6. Attenuation spectra for Versamag: The solid line is the raw experimental data, the dashed line is the initial third order polynomial fit, and the dotted line is the attenuation function fitted in the sizing process.

variable. The results are shown in Table IV, which indicates that the free density result returned a slightly smaller median size and both procedures returned a slightly reduced standard deviation. Figure 5 shows the resulting PSDs expressed as distributions on logarithmic abscissae in which the difference between the two calculated results was barely significant.

It is of interest to investigate the quality of the fit to the measured attenuation data. Figure 6 shows the superposition of the relevant attenuation spectra-the measured data, the third order fit to these, and the attenuation function which resulted from the fitting process. It is clear that all three are in good agreement. The approximation to the bimodal distribution by the unimodal distribution obtained ultrasonically is clear.

To illustrate the significance of density, the sizing calculation was repeated for density values set to $\pm 10 \%$ of the true value and the results are compared to the fixed density result from Fig. 5 in Fig. 7. Both of the changed density results diminish the standard deviation; the lower density result reduces the median size whilst the higher density result increases the median size.

We stated earlier that the attenuation calculation was relatively insensitive to all of the parameter values except

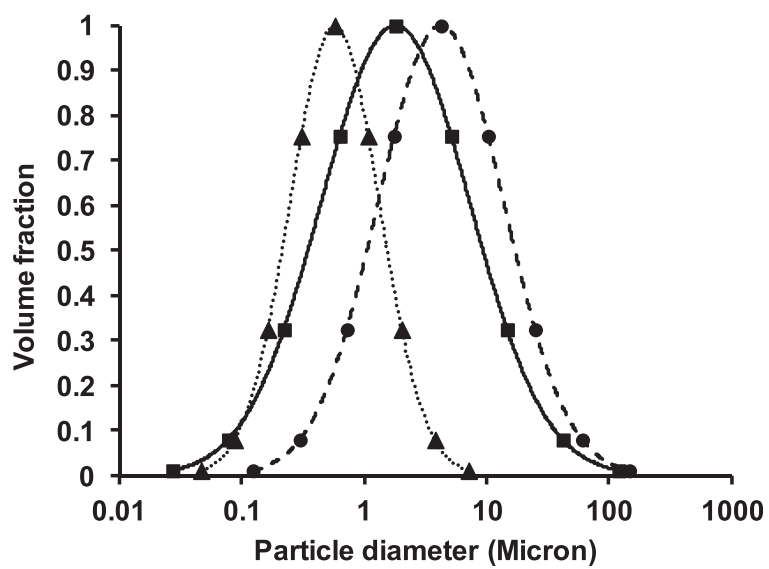

FIG. 7. PSDs of Versamg with the correct density (solid line), $+10 \%$ density change (dashed line), and $-10 \%$ density change (dotted line).

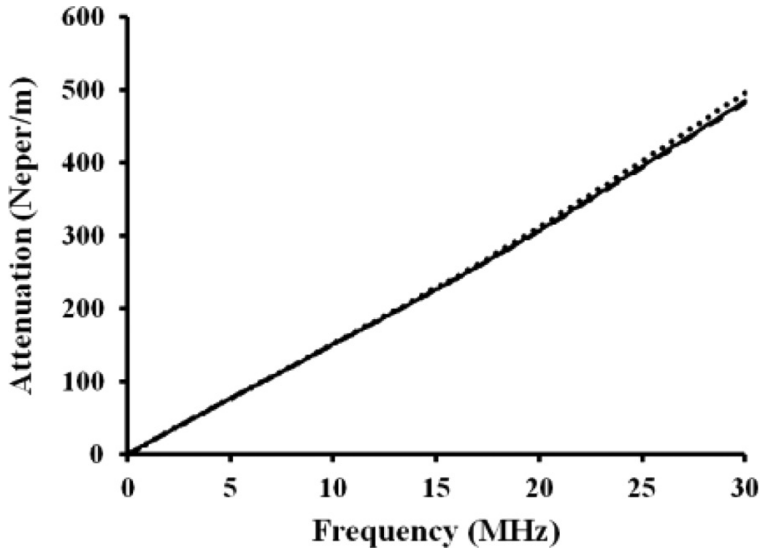

FIG. 8. Attenuation spectra for Versamag: The solid line is the attenuation function obtained during the fitting process; the dashed line represents $+50 \%$ change in all parameters except density (Table II) and the dotted line corresponds to a $-50 \%$ change.

density. To confirm this, Fig. 8 shows the attenuation spectrum fitted in the particle sizing calculation with fixed density and, superimposed, two further curves which were calculated when all of the parameters in Table II, except density, were changed by $\pm 50 \%$. The insensitivity to these parameters is clear.

Finally, we investigate how the fitted PSD might have changed had there been $\pm 10 \%$ errors in the attenuation measurement. The results in Fig. 9 show that the changed attenuation has significantly affected the PSDs; the $10 \%$ increase in attenuation has reduced the median by $60 \%$ and the $10 \%$ decrease has increased the median by $60 \%$. The standard deviations (widths) have not changed significantly. The trend in these results is not unexpected on the basis that a lower median value implies a larger number of smaller scattering particles and, hence, increased attenuation, and vice versa. The results confirm the need for accurate and precise attenuation measurements.

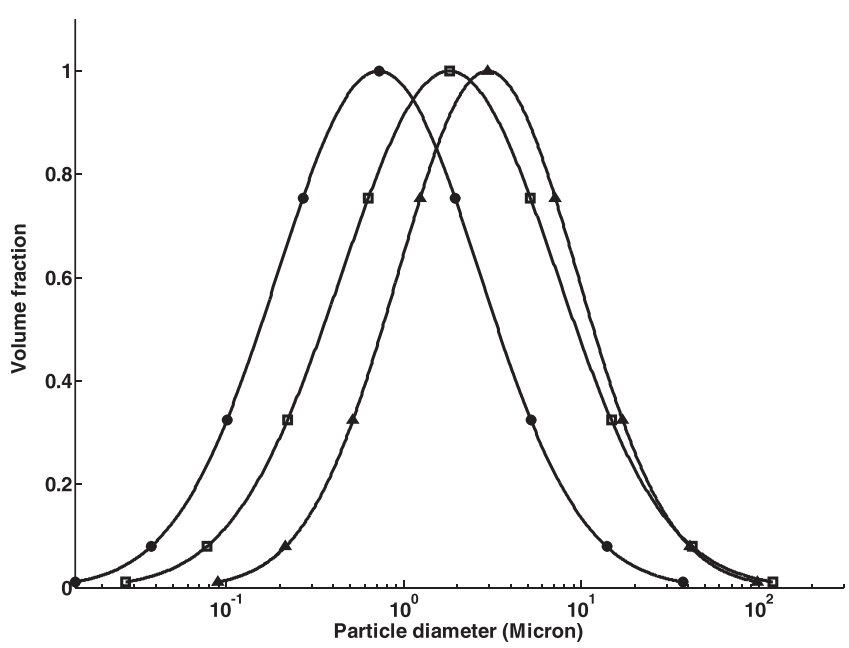

FIG. 9. Calculated PSDs for Versamag: The fitted PSD from Fig. 5 (squares), PSD calculated when attenuation is increased by $10 \%$ across the frequency band (circles), and when attenuation is decreased by $10 \%$ across the band (triangles). 


\section{Polydisperse case 2: Glass beads}

In the experiments described so far, the dispersed particle sizes were relatively small $-30 \mathrm{~nm}$ for silica particles and $1.8 \mu \mathrm{m}$ for Versamag. It is of interest to test the method on larger particles and, to this end, we have used some historic Mastersizer (Malvern Instruments Ltd., Malvern, UK) data from a $0.27 \% \mathrm{v} / \mathrm{v}$ suspension of glass beads with a median size $35.6 \mu \mathrm{m}$ and standard deviation 0.35 . The corresponding attenuation data were not available so the expected attenuation was calculated using the extended ECAH model, but using the viscosity value of water for the continuous phase on account of the low concentration. The density value was $2500 \mathrm{~kg} \mathrm{~m}^{-3}$. The particle sizing procedure was applied to the calculated attenuation with density fixed and as a free variable. The calculated PSDs are shown with the Mastersizer (Malvern Instruments Ltd., Malvern, UK) result in Fig. 10 and the numerical results are given in Table V. Clearly, there is good agreement between the results; we expect that had ultrasonic attenuation data been available, an equivalent agreement would have been obtained.

\section{Polydisperse case 3: Simulated high mass density}

In the experiments described in Secs. V A, VB, and $\mathrm{V} \mathrm{C}$, the disperse phase materials all exhibited densities close to that of silica. A study using materials of greater density would clearly be of interest here, particularly, with relatively large particle sizes so as to represent typical mineral processing situations. We have performed such a study using computational modeling rather than experiment because we did not have available appropriate suspensions that were stable against sedimentation. The Mastersizer (Malvern Instruments Ltd., Malvern, UK) PSD from Fig. 10 and the glass bead data from Table I have been used as the basisexcept density, which has been raised to $5000 \mathrm{~kg} \mathrm{~m}^{-3}$. The attenuation as a function of frequency was then calculated to give a simulated "measured" attenuation function. The particle sizing calculation was then performed with density as either a fixed or free variable, as before. The resulting PSD is shown in Fig. 11 and the associated numerical parameters in Table VI; the similarity with the original data is clear.

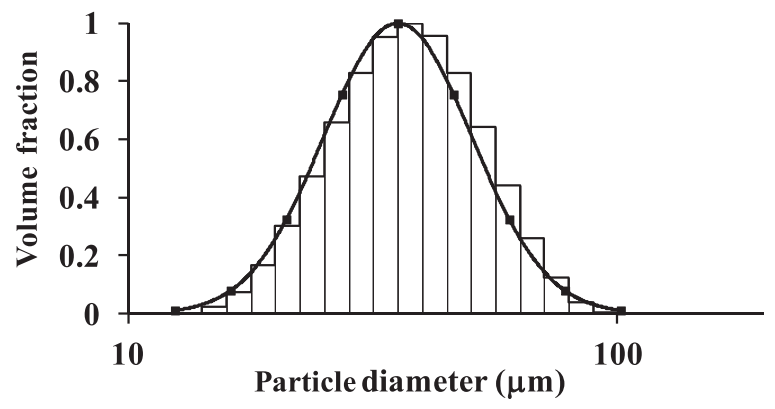

FIG. 10. PSDs for a $0.27 \% \mathrm{v} / \mathrm{v}$ aqueous suspension of glass beads with median size $35.6 \mu \mathrm{m}$ and standard deviation 0.35 . The histogram is the Mastersizer (Malvern Instruments Ltd., Malvern, UK) result, the solid line is the PSD obtained with fixed density, and the squares represent the fitted PSD with density as a free variable.
TABLE V. PSD parameters of the glass bead sample obtained with the Mastersizer (Malvern Instruments Ltd., Malvern, UK), and ultrasonically with either a fixed value of density or with density as a free variable in the fitting process.

\begin{tabular}{lccc}
\hline \hline PSD type & Median $(\mu \mathrm{m})$ & Standard deviation & Density $\left(\mathrm{kg} \mathrm{m}^{-3}\right)$ \\
\hline Mastersizer & 35.6 & 0.35 & $\mathrm{n} / \mathrm{a}^{\mathrm{a}}$ \\
Fixed density & 35.6 & 0.35 & 2500 \\
Free density & 35.6 & 0.35 & 2484 \\
\hline \hline
\end{tabular}

an/a: Not applicable.

\section{DISCUSSION AND CONCLUDING REMARKS}

We have shown that computations of ultrasonic attenuation in solid-in-liquid suspensions with relatively small particles are only weak functions of all of the physical properties of the solid phase, except density. This implies that an accurate value for density should, ideally, be known a priori for successful PSD estimation using ultrasonic wave attenuation as its basis. However, in many instances, the material density will not be known and may be difficult to measure, for example, when such measurements are associated with significant hazard. This paper has demonstrated that an accurate estimate of PSD can be obtained by allowing the solid phase density to be a free variable in the fitting process. On the basis of earlier work, for solid phase concentrations $z 2 \% \mathrm{v} / \mathrm{v}$, we have used the Happel formulation for the viscosity of the continuous phase in the ECAH model, which forms the basis of the PSD estimation. This can be regarded as a pragmatic approximation which can be used up until the time when a more thorough theoretical approach might be available.

The results for the monodisperse suspension of small silica particles (Ludox, Table III) with density as a free variable indicated that the accuracy of the size estimate improved slightly at the highest concentration $(8.22 \% \mathrm{v} / \mathrm{v})$; we do not attribute any significance to this as all three results were within $4 \%$ of the nominal size $(30 \mathrm{~nm})$. For the first polydisperse case (Versamag, Table IV), the free density result was slightly different from the nominal median and standard deviation, but the difference was insignificant when observed graphically in log-normal space (Fig. 5). We note also here that the PSD obtained ultrasonically was a single mode approximation to a bimodal distribution obtained

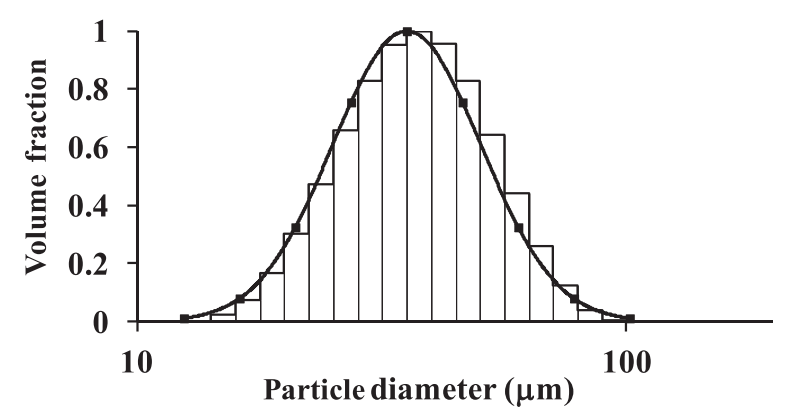

FIG. 11. PSDs for the high mass density experiment. The histogram is the distribution used to simulate "measured" attenuation, the solid line is the PSD obtained with fixed density, and the squares represent the PSD obtained with density as a free variable. 
TABLE VI. PSD data for the simulated high mass density experiment.

\begin{tabular}{lccc}
\hline \hline PSD type & $\begin{array}{c}\text { Median } \\
(\mu \mathrm{m})\end{array}$ & $\begin{array}{c}\text { Standard } \\
\text { deviation }\end{array}$ & $\begin{array}{c}\text { Density } \\
\left(\mathrm{kg} \mathrm{m}^{-3}\right)\end{array}$ \\
\hline $\begin{array}{l}\text { Mastersizer (Malvern } \\
\text { Instruments Ltd., Malvern, UK) }\end{array}$ & 35.6 & 0.35 & $\mathrm{n} / \mathrm{a}^{\mathrm{a}}$ \\
$\begin{array}{l}\text { Fixed density } \\
\text { Free density }\end{array}$ & 35.6 & 0.35 & 5000 \\
\hline \hline
\end{tabular}

${ }^{\mathrm{a}} \mathrm{n} / \mathrm{a}$ : Not applicable.

optically. The sensitivity of the PSD to the density value was demonstrated in Fig. 7, which indicated that a $10 \%$ change in the assumed density would shift the median size by as much as $70 \%$. Similarly, an error in the broadband attenuation measurement of $10 \%$ was shown to change the estimate of the median particle size by $\sim 60 \%$, confirming the generally accepted requirement for accurate and precise measurements of attenuation, as well as a robust procedure for fitting the attenuation spectrum based on a propagation model such as ECAH. We have shown that the measured and fitted attenuations were very close to each other in the Versamag experiment (Fig. 6). In principle, a formal error analysis would be of advantage here; it would follow our methods in Ref. 17 and, consequently, would be lengthy and beyond the scope of this paper. We have asserted that, apart from density, the physical properties of the disperse phase have little effect on the calculated attenuation and we have demonstrated this clearly in Fig. 8. We would conclude, at this point, that the proposed technique appears to be robust and, in addition to PSD estimation, provides a means to measure the density of particulate materials.

The Ludox and Versamag experiments were associated with relatively small particles and low densities close to that of silica. It was therefore of interest to consider much larger particles and particles of much greater density. We did not have experimental data for either of these cases and so they were addressed in a simulation exercise; this showed that the technique worked well in both cases (Figs. 10 and 11) and, notwithstanding the limitations of modelled results, we would assert that they provide evidence that the technique can be applied in these cases.
${ }^{1}$ R. E. Challis, M. J. W. Povey, M. L. Mather, and A. K. Holmes, "Ultrasound techniques for characterizing colloidal dispersions," Rep. Prog. Phys. 68(7), 1541-1637 (2005).

${ }^{2}$ P. S. Epstein and R. R. Carhart, "The absorption of sound in suspensions and emulsions. I. Water fog in air," J. Acoust. Soc. Am. 25(3), 553-565 (1953).

${ }^{3}$ J. R. Allegra and S. A. Hawley, "Attenuation of sound in suspensions and emulsions: Theory and experiments," J. Acoust. Soc. Am. 51(5B), 1545-1564 (1972).

${ }^{4}$ BS ISO 20998-1, Measurement and characterization of particles by acoustic methods-Part 1: Concepts and procedures in ultrasonic attenuation spectroscopy (British Standards Institute, London, 2012), p. 30.

${ }^{5}$ BS ISO 20998-2, Measurement and characterization of particles by acoustic methods-Part 2: Guidelines for linear theory (British Standards Institute, London, 2012).

${ }^{6}$ G. W. C. Kaye and T. H. Laby, Tables of Physical and Chemical Constants, 16th ed. (Longman, Harlow, 1995), p. 144.

${ }^{7}$ D. R. Lide, CRC Handbook of Chemistry and Physics: A Ready-Reference Book of Chemical and Physical Data, 91st ed. (CRC, London, 2010), p. 2610.

${ }^{8}$ R. S. Al-Lashi and R. E. Challis, "Uncertainties in ultrasonic particle sizing in solid-in-liquid suspensions," IEEE Trans. Ultrason. Ferroelectr. Freq. Control 61(11), 1835-1845 (2014).

${ }^{9}$ R. S. Al-Lashi and R. E. Challis, "Effective viscosity in a wave propagation model for ultrasonic particle sizing in non-dilute suspensions," J. Acoust. Soc. Am. 136(4), 1583-1590 (2014).

${ }^{10}$ J. Happel, "Viscosity of suspensions of uniform spheres," J. Appl. Phys. 28(11), 1288-1292 (1957).

${ }^{11} \mathrm{~J}$. Happel, "Viscous flow in multiparticle systems: Slow motion of fluids relative to beds of spherical particles," AIChE J. 4(2), 197-201 (1958).

${ }^{12}$ J. Happel and H. Brenner, Low Reynolds Number Hydrodynamics-With Special Applications to Particulate Media (Prentice-Hall, Englewood Cliffs, NJ, 1965).

${ }^{13}$ P. Lloyd and M. V. Berry, "Wave propagation through an assembly of spheres: IV. Relations between different multiple scattering theories," Proc. Phys. Soc. 91(3), 678-688 (1967).

${ }^{14}$ R. E. Challis, J. S. Tebbutt, and A. K. Holmes, "Equivalence between three scattering formulations for ultrasonic wave propagation in particulate mixtures,” J. Phys. D: Appl. Phys. 31(24), 3481-3497 (1998).

${ }^{15} \mathrm{D}$. W. Marquardt, "An algorithm for least-squares estimation of nonlinear parameters,” J. Soc. Ind. Appl. Math. 11(2), 431-441 (1963).

${ }^{16} \mathrm{D}$. W. Marquardt, "Solution of nonlinear chemical engineering models," Chem. Eng. Prog. 55(6), 65-70 (1959).

${ }^{17}$ A. N. Kalashnikov and R. E. Challis, "Errors and uncertainties in the measurement of ultrasonic wave attenuation and phase velocity," IEEE Trans. Ultrason. Ferroelectr. Freq. Control 52(10), 1754-1768 (2005).

${ }^{18}$ V. A. Del Grosso and C. W. Mader, "Speed of sound in pure water," J. Acoust. Soc. Am. 52(5B), 1442-1446 (1972).

${ }^{19}$ M. C. Smith and R. T. Beyer, "Ultrasonic absorption in water in the temperature range $0^{\circ}-80^{\circ} \mathrm{C}$," J. Acoust. Soc. Am. 20(5), 608-610 (1948). 